\title{
The effects of practice with one positive set in a memory scanning task can be completely transferred to a different positive set
}

\author{
MARIANNE W. KRISTOFFERSON \\ Department of Psychiatry, McMaster University, Hamilton, Ontario, Canada
}

\begin{abstract}
Recognition tasks in which the items have been assigned to one and only to one response category throughout an experiment typically reveal that the functions relating reaction time and positive set size are negatively accelerated and have slopes which decrease significantly with practice. The present experiments were designed to determine whether this practice effect is specific to the individual items practiced or if it depends, at least partially, on relations built up among the positive set items. The results permit rejection of both alternatives. In the extreme case tested, it was found that, after prolonged practice with the same items composing nested positive sets, these items could be replaced with unpracticed ones without producing any significant change in the effect of set size, reaction times, or error levels. Absence of change in overall reaction times in combination with stability of slope values leads to the conclusion that not only do those practice effects which are related to the set-size effect transfer exceedingly well to unpracticed items, but practice effects on all other stages involved in item recognition also show almost total transfer to unpracticed items. It is concluded that, under the conditions of these experiments, the gain (through practice) in efficiency in processing the stimulus is independent of the stimulus.
\end{abstract}

In a typical item-recognition task (e.g., Sternberg, $1966,1969)$, the subject must respond "yes" or "no," usually by pressing the appropriate one of two response keys, to indicate whether a visually presented test item is or is not a member of a predefined set of positive items. The size of the positive set is varied and response latencies are recorded.

A number of studies have shown that when the construction of the item-recognition task is such that a given item in the stimulus set is assigned to one and only to one response category over all trials (response consistency), then the findings differ in important ways from those obtained when a given item in the total stimulus set sometimes requires a positive response and sometimes requires a negative response (response inconsistency). (For examples of response-consistency designs, see Briggs \& Blaha, 1969; Kristofferson, 1972b; Ross, 1970. For examples of response-inconsistency designs, see Kristofferson, 1972a; Sternberg, 1966, 1969.) Differences between the two versions of the task are most pronounced when the effects of practice on performance are examined.

When the response-inconsistency construction of the task is used, it is frequently reported that the function relating response latencies and positive set size (the

This study was supported by Grant A8620 from the National Research Council of Canada. I thank Rose Silbert and Nancy Silbert for their assistance with data collection and analyses. I thank the Medical Research Council, Applied Psychology Unit at Cambridge, England, for its hospitality during the period when this paper was written. And I thank Alfred Kristofferson for many fruitful discussions about this study. item-recognition function) is well described as linear, and that the slopes of the item-recognition functions are the same whether calculated from trials requiring a positive response or from trials requiring a negative response. Typically, when digits or letters are the stimuli, item-recognition time is increased by approximately $35-40 \mathrm{msec}$ for each additional item assigned to the positive set (Kristofferson, 1972a; Sternberg, 1966, 1969). For this construction of the task, it has also been shown that practice does not alter the effects of positive set size (Burrows \& Murdock, 1969; Kristofferson, 1972a; Nickerson, 1966). Itemrecognition functions remain linear, positive and negative slopes do not differ significantly, and slope values remain constant over the course of prolonged practice. It should be noted that, when practiced performance has been studied for this construction of the task, not only has there been response inconsistency between different set-size conditions within a session (since items which require a positive response for a given set-size condition require a negative response for all other set-size conditions), but also a new assignment of the items from the total stimulus set to positive, and thus negative, sets has been made for each session.

On the other hand, the response-consistency construction of the item-recognition task has repeatedly yielded evidence of nonlinear item-recognition functions which are nearly always negatively accelerated, and sometimes well fit by linear functions when reaction time (RT) is plotted against the log of the positive set size. The slope of the obtained functions has generally been found to decrease significantly over the course of practice (Briggs \& Blaha, 1969; Kristofferson, 1972b; 
Ross, 1970; Simpson, 1972). In all of the cited studies, response consistency existed both within and between sessions and the assignment to response category of the items making up the total stimulus set was fixed and constant over all sessions. It is the nature of the practice effect in this particular construction of the task that is investigated and reported here.

From the Kristofferson (1972b) study, in which the effect of prolonged practice upon the effect of positive set size on item-recognition time was investigated for the response-consistency construction of the task, the findings of interest were: (1) The item-recognition function was found to be not linear, but negatively accelerated; (2) the amount by which RT was increased by the addition of a given number of items to the positive set (the set-size effect) decreased significantly with practice for both negative and positive trials; and (3) throughout the course of practice, the set-size effect was significantly greater for positive than for negative trials.

A general theoretical framework consistent with these results was proposed, which had as its basic assumption that when specific and prolonged practice on positive and negative set items is provided under conditions of response consistency, features which uniquely define each positive set are extracted and organized into a hierarchy of tests. The tests are then made in sequence until enough information is obtained to determine whether the test item is a member of the positive set, at which point they terminate.

Both Ross (1970) and Simpson (1972) have also suggested that item-recognition performance, when investigated under the procedure outlined above, may be explained by assuming that features which characterize more than one item in a given positive set are defined and used in the decision process.

Graboi (1971) has offered a similar explanation for practice effects obtained in some visual search experiments in which, after extended practice with constant assignment of items to target sets, there remained a small effect of memory set size upon search time, and the function relating the two variables was negatively accelerated. Graboi attributed the practice effects to the selective development of those feature analyzers relevant to each target set.

The most obvious alternative explanation of the effects of practice on the set-size effect would be one which assumed that the comparison process is altered through: (1) effects on each individual positive item only, or (2) effects on each individual item in combination with effects across each positive set of items.

Experiment 1 was designed to test among these alternatives, and to determine whether the practice effect is specific to the individual items practiced, or whether it depends, at least partially, on relations built up among the items in the positive sets.

In Experiment 1, the response-consistency construction of the item-recognition task was used. Subjects were provided with an equal amount of prolonged practice on two nonoverlapping sets (" $A$ " and " $B$ ") of positive items, where, from each set, positive sets of Sizes 2, 4, and 6 were formed. During the practice phase, subjects alternated daily between working with Set A and Set B. Negative sets were composed of the same items for all sessions. Following the practice sessions, two additional sessions were run, during which some subjects continued to work with Sets $A$ and $B$, as during the practice sessions (old group), some subjects worked with positive sets composed half of items from Set A and half of items from Set B (old mixed group), and a third group of subjects worked with positive sets composed half of items previously practiced, from Set A or B, and half of new items never previously used as positive or negative items (old-new mixed group).

Assuming the previously reported practice effects are obtained in the present study, comparisons between the three groups should allow selection among the alternatives suggested. If the practice effect is confined to the individual items practiced, then the change in performance, if any, for the old mixed group from the final 2 days of practice to the two subsequent test sessions (change scores) should not differ significantly from that shown by the old group. Further, the set-size effect for the old-new mixed group should increase significantly relative to the other two groups. If the practice effect is dependent only upon the relations built up across the positive set items, the set-size effect should increase significantly for the old-mixed group relative to the old group when change scores are compared. Similarly, for the old-new mixed group, the set-size effect should increase relative to the old group when change scores are compared. Should the practice effect be partially attributable to the specific items and partially to relations built up over groups of items, then a comparison of change scores should result in showing a significantly larger set-size effect for the old-new mixed group than for either the old mixed or old group; and, further, the old mixed group should show a significantly larger set-size effect than the old group.

\section{EXPERIMENT 1}

\section{Method}

Apparatus and stimulus materials. A Scientific-Prototype three-field tachistoscope was used for the presentation of the stimulus materials and the control of trial events. Stimulus materials were the letters of the English alphabet (black Letraset Franklin Gothic, $1.59 \mathrm{~cm}$ high), which were individually centered on white cards. The visual angle subtended was approximately $1.25 \mathrm{deg}$.

Subjects. The subjects were male volunteers from the student body of McMaster University. They were paid $\$ 2$ per session, and were all naive to the experimental task.

Design. Each of the 18 subjects were run individually in 26 daily experimental sessions, excluding weekends. Each session had three parts, one for each of the positive set sizes $(2,4$, and 6). There were two successive blocks of 24 trials for each of the set-size conditions. One-minute breaks occurred between blocks of trials, and 2-min breaks between set-size conditions. Nine 
practice trials preceded collection of data on each set-size condition. On each trial a centered black dot, which served as both a warning signal and a fixation point, was first displayed for $1.5 \mathrm{sec}$. The dot was immediately replaced by a letter which was displayed for $44 \mathrm{msec}$. A blank white field was then displayed for the remainder of the 10 -sec interval. The time from the onset of the test stimulus letter to the subject's response was recorded with an accuracy of $\pm 1 \mathrm{msec}$. An auditory signal was used to provide accuracy feedback on each trial.

Six subjects were randomly assigned to each of the three groups: old, old mixed, and old-new mixed. For each subject, each of the six possible orders of the three set-size conditions was administered over every six consecutive sessions. The order within each 6-day block was determined randomly for each of the six trios of subjects separately (one subject being a member of each group), with the restriction that, for a given session, each subject in each group had a different one of the six possible orders.

During Sessions 1-24, all subjects in all groups obtained practice under those item-recognition procedures which have previously yielded evidence of: (1) negatively accelerated itemrecognition functions, (2) a decrease in the slope, or difference scores, over the course of practice, and (3) difference scores, or slopes, which are significantly smaller for negative than for positive trials. During these sessions, each subject practiced with nested positive sets of Sizes 2,4 , and 6 , in which the items comprising the sets were different and nonoverlapping for even-numbered and odd-numbered sessions. This procedure made possible the manipulations of set structures for each of the groups in the second phase of the experiment (Sessions 25-26) needed to determine whether the practice effect is set specific or item specific.

Sessions 1-24: Assignment of stimuli to sets. For each trio of subjects separately (one subject in each of the old, old mixed, and old-new mixed groups), two sets of positive items (Set A and Set B), composed of six letters each, were randomly selected without replacement from the 26 letters of the English alphabet. Positive sets of Sizes 2, 4, and 6 were constructed separately from items within Set A and Set B so as to provide nested and constant positive sets. The letters composing the positive sets were never organized as a pronounceable word or nonword. On odd-numbered sessions, subjects worked with the three positive set sizes constructed from the items in Set $A$, and, on even-numbered sessions, subjects worked with the three positive set sizes constructed from the items in Set B. Negative items for each trio of subjects were 8 letters drawn randomly from the 14 letters remaining in the pool after the two sets of positive letters had been drawn for that trio of subjects. The same eight negative items composed the negative set for all set sizes and all sessions. Thus, within a given set (A or B) condition, nested and constant positive sets were used; both within and across set conditions, response consistency existed.

Sessions 25-26: Assignment of stimuli to sets. Items assigned to the negative set remained the same as in all previous sessions for all subjects in all groups.

For subjects in the old group, for Session 25, positive sets of Sizes 2, 4, and 6 were composed of the same items as were used for all previous odd-numbered sessions; and, for Session 26, positive sets of Sizes 2,4 , and 6 were composed of the same items as were used for all previous even-numbered sessions.

For subjects in the old mixed group, for Session 25, each of the positive set sizes $(2,4$, and 6$)$ were constructed half of items previously assigned to those set sizes on even-numbered sessions and half of items previously assigned to those set sizes on oddnumbered sessions. Similarly, for Session 26, each of the positive set sizes $(2,4$, and 6$)$ were constructed half of items previously assigned to those set sizes on even-numbered sessions and half of items previously assigned to those set sizes on odd-numbered sessions, such that there was no overlap in items used in Sessions 25 and 26.
For subjects in the old-new mixed group, for Session 25 , each of the positive set sizes were constructed half of items previously assigned to those set sizes on odd-numbered sessions, and half of items which were "new," that is, letters not previously used as members of either positive or negative sets of items. In Session 26, each of the positive set sizes were constructed half of items previously assigned to those set sizes on even-numbered sessions, and half of new items.

All sessions. In each session (1-26), before each part of the experiment, the subject was handed a card showing the letters which formed the positive set for that part. He was instructed to press the key under the control of his preferred hand whenever any one of the letters on the card appeared and to press the key under the control of his nonpreferred hand whenever any other letter appeared. He was told to respond as quickly as possible without making mistakes.

For all sessions and for each of the set-size conditions, 50\% of the trials required a positive response. With that restriction, a set of twelve different random orderings of positive and negative trials was made up separately for each of the set-size conditions. The random orderings were then assigned to each trio of subjects over sessions so that a particular ordering was used, at most, three times. Individual items composing both the positive sets and the negative set were presented randomly, with the restriction of equal frequency of occurrence.

\section{Results and Discussion}

Reaction times to practice trials were deleted from all analyses, as were reaction times on all trials in which the incorrect key was pressed.

Analyses of set-size effects during practice. The data collected over the first 24 days of the experiment were first analyzed to ascertain: (1) whether the features previously found (Kristofferson, 1972b) to describe data from a response-consistency construction of an itemrecognition task were replicated, and (2) whether the important features of the data were the same for all three groups of subjects. More specifically, analyses were carried out to determine for each group: (1) whether the function relating positive set-size and itemrecognition time (reaction time) was negatively accelerated, (2) whether the positive set-size effect diminished over the course of practice, and (3) whether the positive set-size effect was greater for positive response trials than for negative response trials.

Through a series of analyses, ${ }_{1}^{1}$ it was first shown that the form of the functions relating reaction time and positive set size was not linear, but was negatively accelerated; and, then, that item-recognition functions were well described as linear when reaction time was plotted against $\log _{2}$ of positive set size.

Slope values were therefore calculated for the loglinear functions, and the primary questions relating to the effects of practice on the parameters of interest were addressed using these measures. Slopes of the functions relating response time to $\log$ of positive set size were determined separately for each subject, for each successive 2-day block, and for positive and negative response trials.

Slope values were analyzed by a three-factor mixeddesign analysis of variance with repeated measures on 
two factors, where groups was the between factor and the within factors were: (1) successive 2-day blocks, and (2) positive and negative response trials. The only significant effect was a main effect of day blocks $[F(11,165)=18.96, p<.0001]$, such that slopes decreased over the course of practice. The magnitude of the effect was large: Slope values averaged approximately $68 \mathrm{msec}$ on the initial 2-day block and decreased to approximately $22 \mathrm{msec}$ by the final 2-day block of practice. The main effect of positive vs negative trials was not significant $[F(1,15)=2.11, \mathrm{p}>.10]$, although the trend was in the direction previously reported by Kristofferson (1972b). From these analyses, it is concluded that the main features found to describe data in the Kristofferson (1972b) study were replicated in the present study.

A mixed analysis of variance was also carried out on the means of the response times ( $\overline{\mathrm{RT}})$ over the 24 practice days. The $\overline{\mathrm{RT}}$ s were averaged over successive 2-day blocks. The between variable was groups, and the within variables were: (1) positive-negative response trials, (2) day blocks, and (3) set size. The main effect of positive-negative response trials was significant $[F(1,15)=165.59, \quad \mathrm{p}<.0001] . \quad$ Throughout the course of practice, and for all groups, negative $\overline{\mathrm{RT}}$ were found to average approximately $50 \mathrm{msec}$ longer than positive $\overline{\mathrm{RT}}$ s. Significant differences between $\overline{\mathrm{RT}}$ s for set sizes were found such that $6>4>2$ for all groups $[\mathrm{F}(2,30)=151.18, \mathrm{p}<.0001]$. There was a significant effect of day blocks $[F(11,165)=29.11$, $\mathrm{p}<.0001]$ and also a significant interaction of Day Blocks by Groups $[\mathrm{F}(22,165)=2.51, \mathrm{p}<.001]$. $\overline{\mathrm{RT}}$ s averaged over all set sizes and over positive and negative trials are plotted against day blocks for each of the groups separately in Figure 1. From inspection of the figure, it is apparent that the old-new mixed group showed a more pronounced decrease in $\overline{\mathrm{RT}}$ over successive day blocks than did other groups. Initially, $\overline{\mathrm{RT}}$ was much greater for the old-new mixed group than for the other two groups, but by the fifth or sixth day block, the $\overline{\mathrm{RT}}$ was approximately equal for all groups. Finally, a significant interaction was found between day blocks and set size $[F(22,330)=10.34$, $\mathrm{p}<.0001]$. Duncan's Multiple Range test showed that with the exception of Day Block 10, on any given day block, $\overline{\mathrm{RT}}$ for Set Size 2 was significantly faster than the $\overline{\mathrm{RT}}$ s for Set Sizes 4 and 6. However, the $\overline{\mathrm{RT}}$ for Set Size 4 was significantly faster than the $\overline{R T}$ for Set Size 6 only on Day Blocks 14. On all other day blocks, while the values of $\overline{\mathrm{RT}}$ were always greater for Set Size 6, the differences were not significant. The course of the change in $\overline{\mathrm{RT}}$ over day blocks was quite similar for all set sizes; the decrease in $\overline{\mathrm{RT}}$ was most marked over the first four or five day blocks; however, $\overline{\mathrm{RT}}$ still showed some decrease over the final two day blocks.

For each subject separately, the percentage of errors obtained was determined for each successive 2-day block and for each positive set size. A mixed analysis of variance resulted in showing a significant main effect of practice $[F(11,165)=5.27, p<.0001]$, a significant main effect of set size $[F(2,30)=16.33, p<.0001]$, and a significant interaction between practice and set size $[F(22,330)=1.99, p<.01]$. There was neither a significant main effect of group, nor any significant interactions with group. Figure 2 shows, for the three groups combined, the percentage of errors obtained, plotted against successive 2-day blocks for each set size separately. With respect to the effects of practice, Duncan's Multiple Range test showed that, for each set size, the percentage of errors obtained on Day Block 1 was greater than for some subsequent

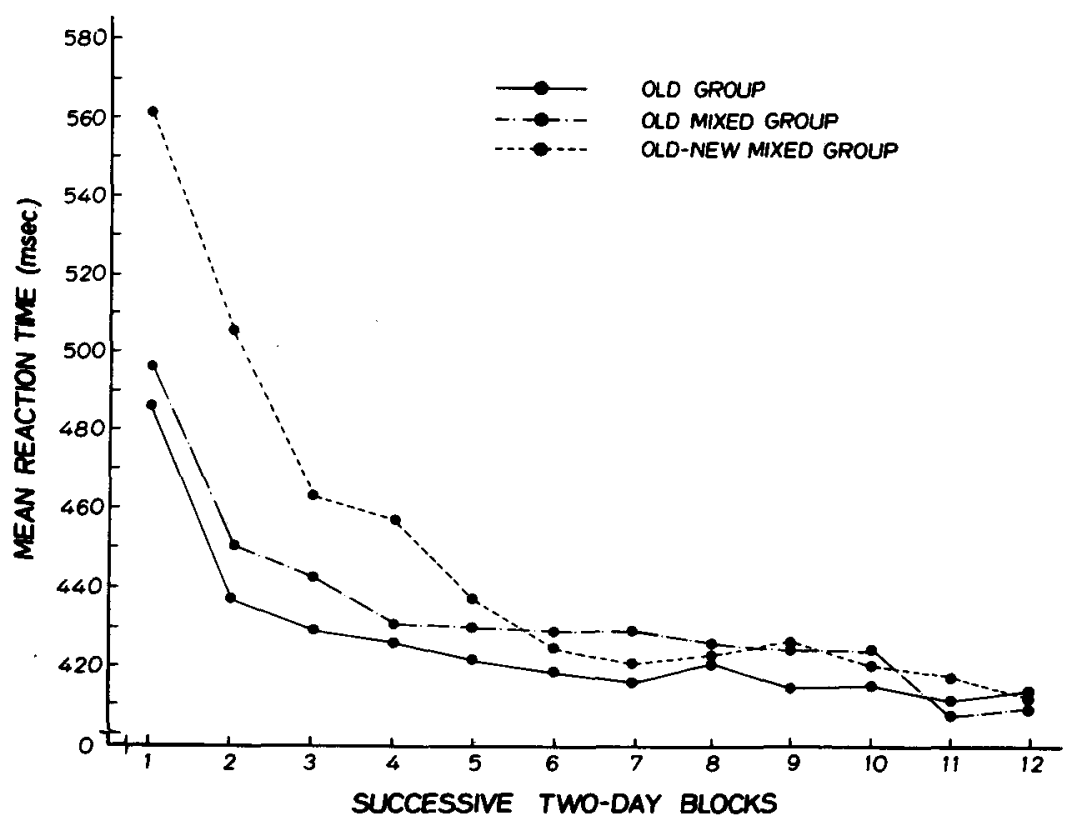

Figure 1. Reaction times averaged over all set sizes and over positive and negative trials shown plotted against successive 2-day blocks for each group separately. 


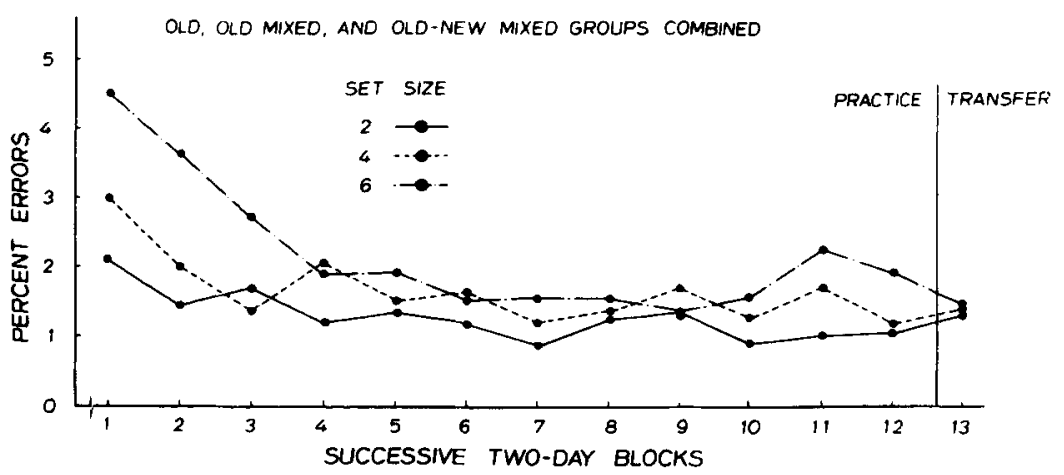

Figure 2. Percent errors obtained over the three groups combined and plotted against successive 2-day blocks for each positive set size separately.

day blocks for that set size. Looking at the set-size effects as shown by the same test, on the first three day blocks, the percentage of errors obtained for Set Size 6 was significantly greater than for Set Sizes 4 and 2. Also, on Day Block 11, errors were significantly greater for Set Size 6 than for Set Size 2. Except for this one departure, by Day Block 4, error levels had stabilized across all set sizes at some value under $2 \%$, and error levels between set sizes did not differ significantly.

Analyses to determine the nature of the practice effect. The analyses concerned directly with the nature of the practice effect were done by combining the data from Days 23 and 24 (Day Block 12: the final days of practice), and comparing those data with the combined data from Days 25 and 26 (Day Block 13: the days on which the construction of positive sets was altered for two of the groups). Separate analyses were carried out on slope values, $\mathrm{RT}$, and errors.

Slope values (slopes of functions relating $\overline{\mathrm{RT}}$ to $\log _{2}$ of set size) were analyzed in a mixed design analysis of variance with the between factor being groups (old, old mixed, and old-new mixed) and within factors: (1) positive-negative response trials, and (2) Day Block 12 vs Day Block 13. None of the main effects were significant. Both the interaction between positive-negative and day blocks $[\mathrm{F}(1,15)=6.75$, $\mathrm{p}<.02]$ and the triple interaction, Positive-Negative by Day Blocks by Groups $[F(2,15)=5.30, p<.02]$ were significant. The latter interaction may be seen in Figures 3, 4, and 5, which show slope plotted against day blocks for positive-negative trials separately for each group. Duncan's tests of the data revealed no given pair of means to differ significantly $(p=.05)$.

A mixed-design analysis of variance was run on $\overline{\mathrm{RT}}$, with the between factor groups and the within factors: (1) positive-negative trials, (2) Day Block 12 vs Day Block 13 , and $(3)$ set size $(2,4,6)$. Three significant effects were found. There was a significant effect of set size $[F(2,30)=34.68, p<.001]$, with $S=6>S=4>S=2$. The main effect of positive vs negative response trials was significant $[F(1,15)=142.64, p<.0001]$ and a significant interaction was found between positivenegative and day blocks $[F(1,15)=4.91, p<.05]$. The positive $\overline{\mathrm{RT}}$ for Day Block 12 was $390.25 \mathrm{msec}$; for Day Block 13, 391.38 msec. The analogous negative $\overline{\mathrm{RT}}$ were $433.67 \mathrm{msec}$ and $441.98 \mathrm{msec}$. For both day blocks, negative $\overline{R T}$ s were significantly longer (by 40-50 msec) than were positive RTs. Duncan's Multiple Range test also showed the increase in $\overline{\mathrm{RT}}$ for negative
Figure 3. Slopes relating response time to $\log$ of positive set size shown plotted over successive 2-day blocks for positive and negative trials separately for the old group.

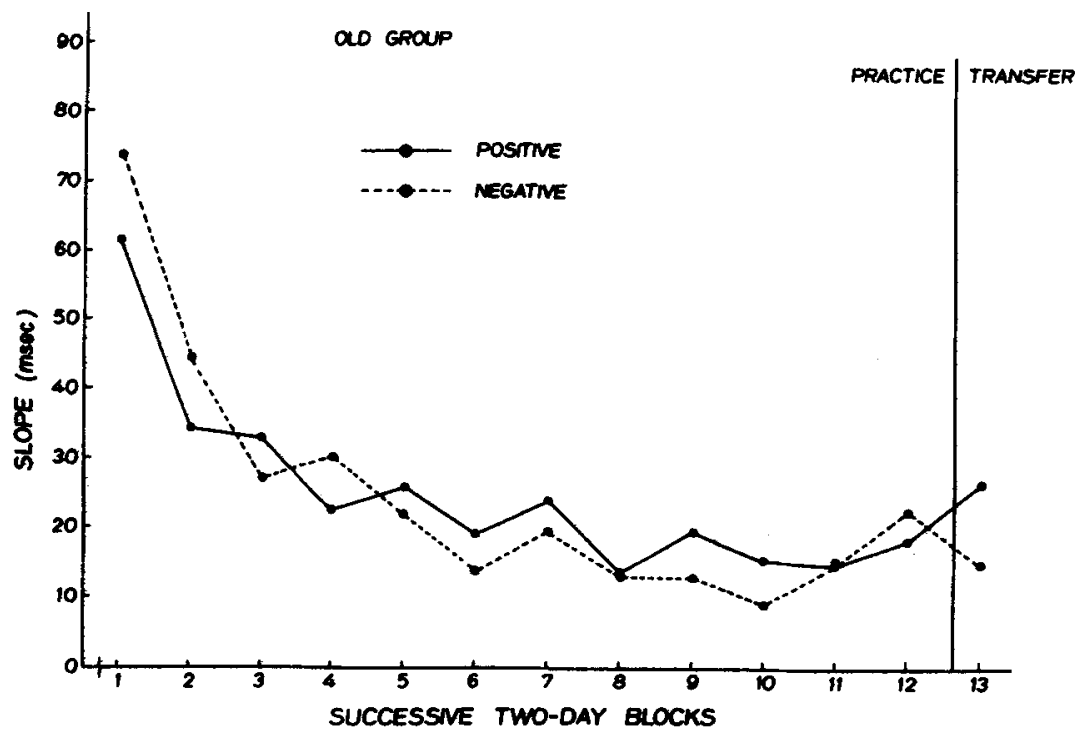




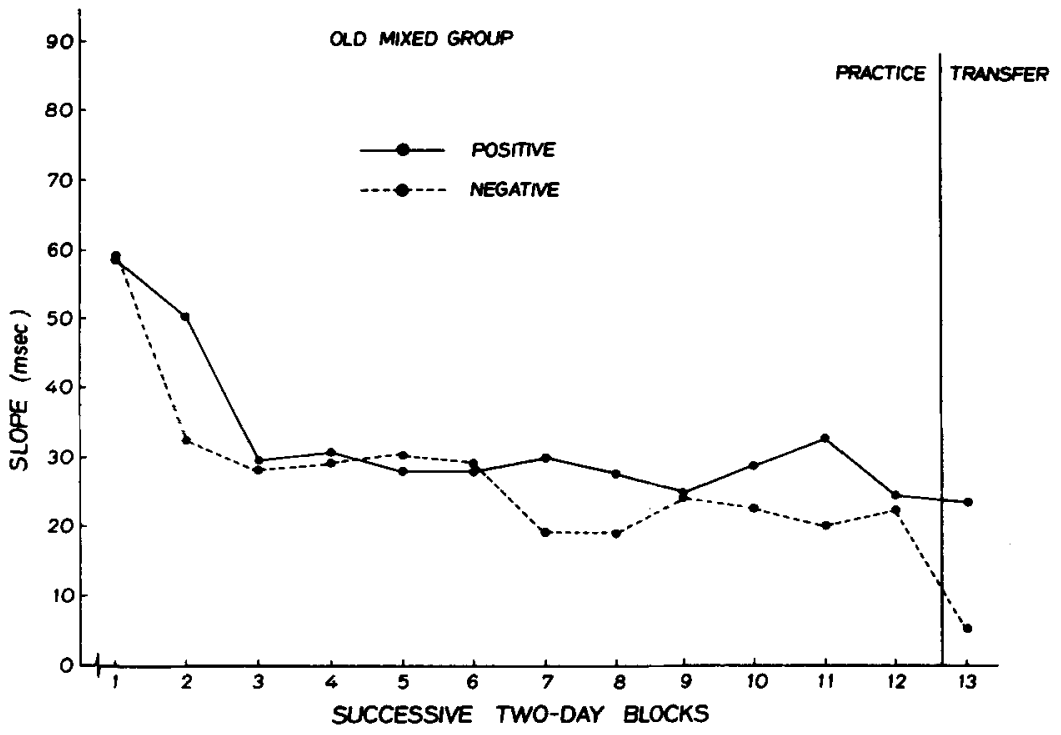

Figure 4. Slopes relating response time to $\log$ of positive set size shown plotted over successive 2-day blocks for positive and negative trials separately for the old mixed group.

trials from Day Block 12 to Day Block 13 to be statistically small, with $\mathrm{p}>.05<.10$. Since there were no significant differences found between groups, the overall conclusion from the analysis must be that the changes in positive set construction were without statistically significant effects on $\overline{\mathrm{RT}}$ s.

A mixed analysis of variance, in which the between and within factors were the same as in the above analysis of $\overline{\mathrm{RT}} \mathrm{s}$, was also carried out on percentage of errors obtained. There were no significant main effects, nor were there any significant interactions. Again, the conclusion drawn is that the experimental manipulations had no statistically significant effect on error levels obtained.

The most striking single finding to appear from all of the analyses was the near absence of effects resulting from the restructuring of the positive sets. Values of slopes, $\overline{\mathrm{RT}}$, and errors obtained by the old mixed group, when the highly practiced positive items were reorganized into never-before-worked-with positive sets, did not show any deterioration to an earlier stage of practice relative to the old group, which continued to work with the highly practiced positive sets. These results argue strongly against any theory which would attribute the practice effect to the development of a hierarchy of features which define each positive set as $\mathrm{a}$ whole and against which the displayed item is tested.

Nor is the hypothesis that the practice effect is due to increased efficiency in the definition of unique features for each item in each positive set tenable in the light of the present data. The performance of the old-new mixed group (those tested with positive sets composed half of practiced items and half of
Figure 5. Slopes relating response time to $\log$ of positive set size shown plotted over successive 2 -day blocks for positive and negative trials separately for the oldnew mixed group.

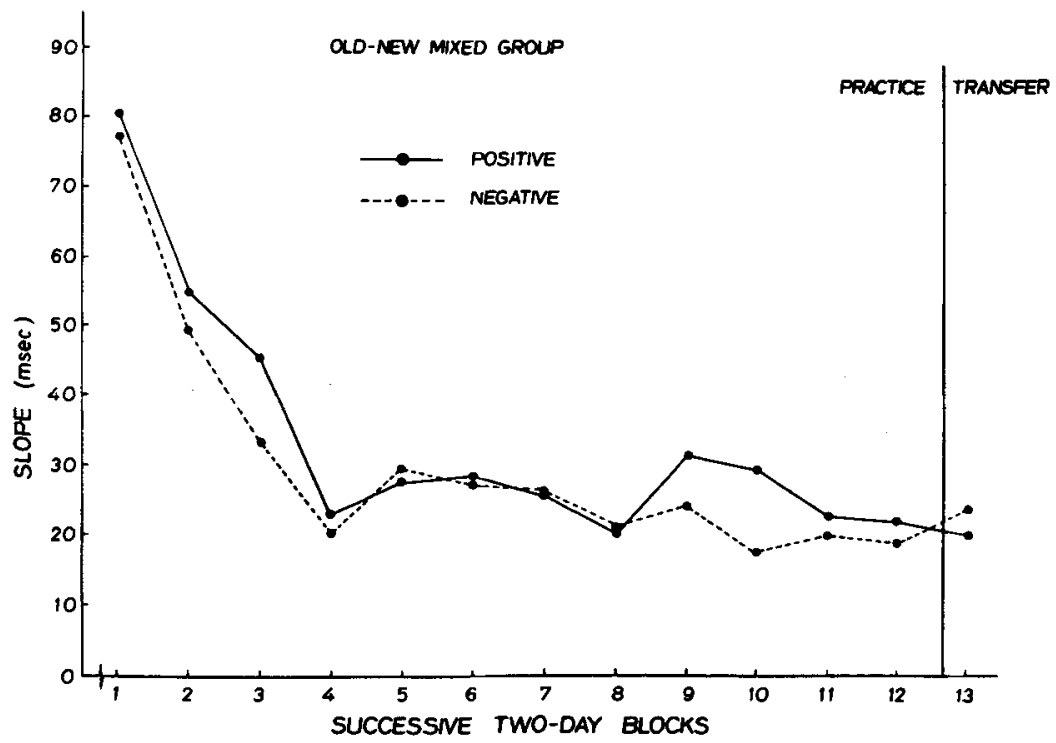


unpracticed items) did not differ significantly on any of the relevant measures from either the old group or the old mixed group. That replacing half the items in highly practiced sets with items which had not been previously practiced failed to disturb the stability of the practice effect is an unexpected and surprising finding. To test whether this finding could be replicated in another and perhaps even stronger test, Experiment 2 was done.

\section{EXPERIMENT 2}

\section{Method and Procedure}

In Experiment 2, six subjects (new group) who were naive to the experimental task, were given 24 days of practice exactly as the three groups in Experiment 1 had been. Then, on Days 25-26, the items composing the positive sets were all replaced by items which had not previously been practiced, as either positive or negative items. Because 20 letters are required for the practice stage of the experiment, it was impossible to provide two different sets of six items for the 2 test days. Therefore, for this group, positive set structures were identical on Days 25 and 26.

\section{Results and Discussion}

The data collected in Experiment 2 were subjected to the same analyses of set-size effects during practice and analyses to determine the nature of the practice effect as those reported for Experiment 1. All previously reported analyses of variance run in Experiment 1 with the three groups as the between factor, were now run with the four groups (old, old mixed, old-new mixed, new) as the between factor.

Analyses of set-size effects during practice. Analyses ${ }^{1}$ revealed for the new group, as they had for the three groups of Experiment 1, that the data were well described as linear when response times were plotted against $\log _{2}$ of positive set size. This result allowed calculation of slope values for these linear functions for subjects in the new group for use in subsequent analyses.

Analysis of slope values by a three-factor mixeddesign analysis of variance was carried out with within factors of successive 2-day blocks and positive and negative response trials. As was found from this analysis in Experiment 1, there was a significant main effect of day blocks $[F(11,220)=26.07, p<.0001]$ and no significant interaction with groups. Slopes decreased over day blocks. The trend for positive slopes to be larger than negative slopes that was reported for Experiment 1 was made significant by the inclusion of the data for the new group in the analysis $[F(1,20)=5.76, p<.05]$. The effect did not interact significantly with groups. The overall mean slope values for positive trials was $30.64 \mathrm{msec}$; for negative trials, $26.87 \mathrm{msec}$.

The results of these analyses allow two conclusions. First, the data obtained from the new group did not differ in any important respects from the data obtained from the three groups in which subjects had been randomly assigned and run some months previously.
Second, the data of Experiment 2 combined with the data from Experiment 1 agree even more closely with findings reported previously (Kristofferson, 1972b) for the same set of procedures, since, in addition to showing that the set-size function was negatively accelerated (logarithmic) and that the effect of positive set size diminished over practice, the finding of significantly greater set-size effects for positive than for negative trials was also replicated.

The analysis of mean reaction times yielded findings which did not differ in any important ways from those reported for Experiment 1. For the new group, values of $\overline{\mathrm{RT}}$ s averaged over positive and negative trials (as are shown in Figure 1 for the other three groups) were initially slightly higher than those for the old mixed group (511 msec), showed the same changes with practice as the other groups, and had, by the final day blocks, become fairly stable at approximately $405 \mathrm{msec}$. Nor did the addition of the new group's data to the error analyses generate any new effects or remove any of the significant effects reported for Experiment 1.

Analyses to determine the nature of the practice effect. As was done in Experiment 1, separate analyses were carried out on slope values, $\overline{\mathrm{RT}}$, and errors, in which comparisons were made between performance on the final 2 practice days (Day Block 12) and on the 2 days when the construction of positive sets was changed for three of the four groups (Day Block 13). In each of the analyses, data from all four of the groups were included.

Analysis of slope values by a three-factor mixed analysis of variance revealed a significant main effect of positive vs negative slopes $[F(1,20)=5.12, p<.05]$ and a significant interaction of Positive-Negative by Day Blocks by Groups $[F(3,20)=8.25, p<.01]$. In Figures 3,4 , and 5 , this interaction is shown for each of the groups in Experiment 1 (old, old mixed, and old-new mixed) as are the slope values obtained over successive day blocks for the entire course of the experiment. In Figure 6, the same aspects of the data are shown for the new group. Duncan's tests applied to the results of the interaction showed only one pair of means to differ significantly $(p<.05)$ : The positive slope value for the new group on Day Block 12 was significantly larger than the negative slope value for the old mixed group on Day Block 13.

Thus, the inclusion of the new group, in addition to boosting the main effect of positive vs negative slopes to significance (which had already appeared in the analyses of practice effects), also generated one statistically significant difference between a pair of means in the triple interaction. The difference is due to the unusually large value of positive slope for the new group (see Figure 6) on the final 2 days of practice (Day Block 12), in conjunction with the unusually small value of negative slope for the old mixed group (see Figure 4) on Day Block 13. By inspection of Figures 3, 4 , and 5 , it is apparent that, for the other groups, over 


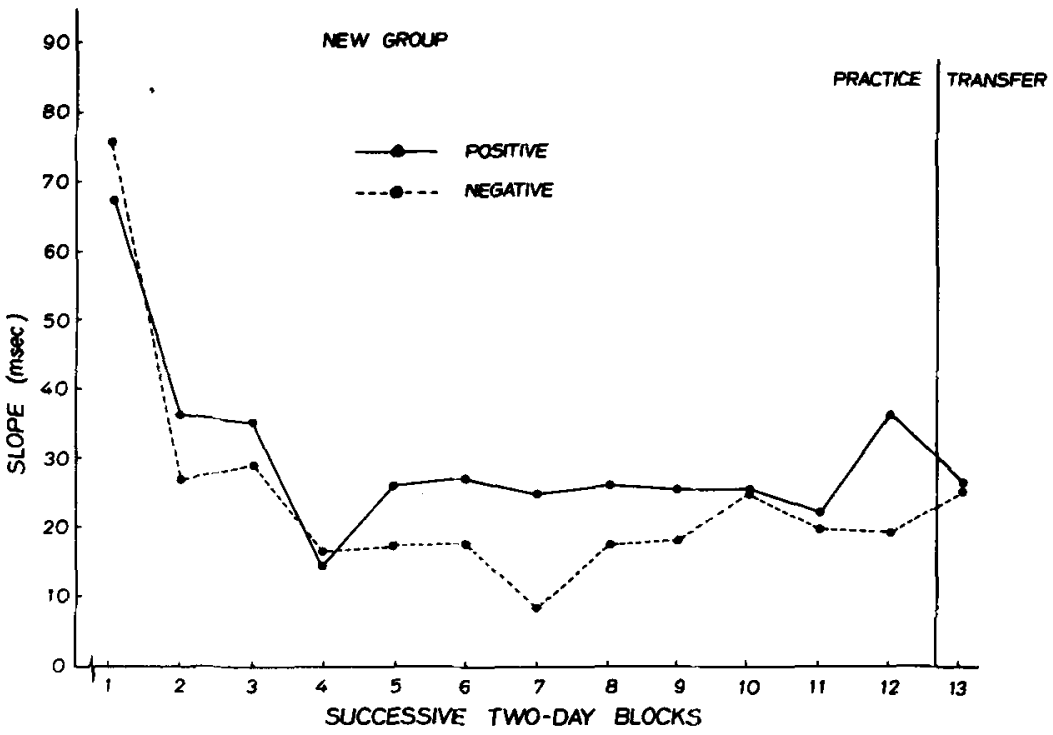

Figure 6. Slopes relating response time to $\log$ of positive set size shown plotted over successive 2-day blocks for positive and negative trials separately for the new group.

the final five or six day blocks during the practice stage, there were perturbations in slopes of equal magnitude to that seen in the new group. If the high slope value on Day Block 12 is considered as a chance perturbation, and the quite stable values of slope for the several preceding day blocks are considered as more representative, then no statistically significant differences between pairs of means would have been found. In any event, replacing the practiced items with all new unpracticed ones did not result in any reversion to an earlier stage of performance by the new group. There is no immediately apparent explanation for the exceptionally small value of slope for negative trials obtained by the old mixed group, especially since it was not accompanied by significant change in mean reaction time or error level. Here, however, it is evident that the direction of change is opposite to that which would be expected if the insertion of some new items into the positive set were to disrupt the effects of practice on the set-size effect.

The analysis of mean response latencies showed the expected main effect of positive vs negative trials $[\mathrm{F}(1,20)=177.17, \mathrm{p}<.0001]$. Positive responses were significantly faster than negative responses for all groups. Similarly, as expected, there was a main effect of set size, with reaction times increasing in duration with set size $[F(2,40)=57.36, p<.0001]$. A main effect was also found for day blocks $[F(1,20)=6.90, p<.02]$, such that overall reaction times on Day Block 13 were significantly greater than on Day Block 12 . The values were $416.63 \mathrm{msec}$ and $410.82 \mathrm{msec}$, respectively. There was no significant interaction of Day Blocks by Groups. Two significant interactions were found: (1) PositiveNegative- by Set Size $[F(2,40)=3.30, p<.05]$, and

Table 1

Positive and Negative RTs and SDs (in milliseconds) for Each Group, Set Size, and Day Blocks 12 and 13

\begin{tabular}{|c|c|c|c|c|c|c|c|}
\hline \multirow[b]{2}{*}{ Group } & \multirow{2}{*}{$\begin{array}{c}\text { Day } \\
\text { Block }\end{array}$} & \multicolumn{3}{|c|}{ Positive Trials by Set Size } & \multicolumn{3}{|c|}{ Negative Trials by Set Size } \\
\hline & & 2 & 4 & 6 & 2 & 4 & 6 \\
\hline & & \multicolumn{6}{|c|}{ Mean Reaction Times } \\
\hline $\begin{array}{l}\text { Old } \\
\text { Old } \\
\text { Old Mixed } \\
\text { Old Mixed } \\
\text { Old-New Mixed } \\
\text { Old-New Mixed } \\
\text { New } \\
\text { New }\end{array}$ & $\begin{array}{l}12 \\
13 \\
12 \\
13 \\
12 \\
13 \\
12 \\
13\end{array}$ & $\begin{array}{l}378 \\
367 \\
360 \\
364 \\
378 \\
388 \\
351 \\
369\end{array}$ & $\begin{array}{l}402 \\
397 \\
387 \\
377 \\
388 \\
393 \\
390 \\
400\end{array}$ & $\begin{array}{l}406 \\
408 \\
399 \\
403 \\
415 \\
423 \\
408 \\
410\end{array}$ & $\begin{array}{l}409 \\
420 \\
419 \\
439 \\
417 \\
431 \\
413 \\
416\end{array}$ & $\begin{array}{l}443 \\
434 \\
441 \\
440 \\
427 \\
450 \\
440 \\
447\end{array}$ & $\begin{array}{l}442 \\
444 \\
455 \\
449 \\
449 \\
470 \\
442 \\
457\end{array}$ \\
\hline New & & \multicolumn{6}{|c|}{ Standard Deviations } \\
\hline $\begin{array}{l}\text { Old } \\
\text { Old } \\
\text { Old Mixed } \\
\text { Old Mixed } \\
\text { Old-New Mixed } \\
\text { Old-New Mixed } \\
\text { New } \\
\text { New }\end{array}$ & $\begin{array}{l}12 \\
13 \\
12 \\
13 \\
12 \\
13 \\
12 \\
13\end{array}$ & $\begin{array}{l}61 \\
46 \\
52 \\
59 \\
60 \\
71 \\
52 \\
57\end{array}$ & $\begin{array}{l}51 \\
52 \\
73 \\
61 \\
63 \\
60 \\
58 \\
64\end{array}$ & $\begin{array}{l}70 \\
66 \\
69 \\
76 \\
66 \\
75 \\
64 \\
64\end{array}$ & $\begin{array}{l}49 \\
62 \\
50 \\
66 \\
54 \\
57 \\
55 \\
54\end{array}$ & $\begin{array}{l}61 \\
52 \\
66 \\
57 \\
61 \\
65 \\
66 \\
62\end{array}$ & $\begin{array}{l}63 \\
51 \\
65 \\
55 \\
58 \\
63 \\
60 \\
54\end{array}$ \\
\hline
\end{tabular}


(2) Positive-Negative by Day Blocks by Set Size by Groups $[F(6,40)=3.35, p<.01]$. In Table 1 , mean reaction times and standard deviations are shown for Day Blocks 12 and 13 for positive and negative trials separately for the new group and for each of the three groups run in Experiment 1.

As was the case for the error analysis in Experiment 1, the analysis of percent errors showed no significant effects when the data from the new group were included. Thus, errors did not change over day blocks, there were no significant differences in error levels for different set sizes, error levels did not differ across groups, and there were no significant interactions between these factors.

\section{GENERAL DISCUSSION}

The findings from Experiment 2 confirm and strengthen the conclusions drawn on the basis of the results obtained in Experiment 1, especially as regards ruling out the possibility that the practice effect is either wholly or partially specific to the individual positive items worked with. To the findings reported from Experiment 1, it can now be added that replacing all of the practiced positive items with unpracticed ones resulted in producing reaction time and error data which did not differ significantly from that obtained from the group in which the practice sessions were simply continued (old group) or from any other group (old mixed and old-new mixed groups). And, this manipulation with the new group produced no increase in the set-size effect relative to the old group or to either of the other two groups run in Experiment 1.

Data from both experiments lead to the rejection of those ideas which attribute the effect of practice on the set-size effect (1) to the relations built up among the items composing the practiced positive sets, or (2) to the individual items which are practiced, or (3) any combination of 1 and 2. To this list can be added any explanation which attributes the practice effect to a change occurring in a hypothetical memory location which is specific to a particular item. Furthermore, the absence of any significant effects on the set-size effect by any of the three experimental manipulations cannot be accounted for by a differential increase in error level with set size, since percent error change scores from the final 2 days of practice to the 2 test days also did not differ significantly from those obtained by the old group.

A more general conclusion to be drawn from these findings is that whatever is learned in the practice stage and is reflected in the changes in the set-size effect over the course of practice is transferred very well indeed to new items under the conditions of this experiment.

Since, for all groups, there were also no statistically significant differences in change scores in mean reaction times, which reflect all stages of processing, it must be concluded that not only do those practice effects on the set-size effect transfer exceedingly well to unpracticed items, but also practice effects on all other stages of processing involved in this construction of the itemrecognition test (encoding, response selection, execution, etc.) also show close to total transfer to unpracticed items.

It is concluded that, under conditions of response consistency and nested and constant assignment of items to positive and negative sets, the gain (through practice) in efficiency in processing the stimulus is independent of the stimulus. Whether this result will obtain for transfer to items of another category, such as digits, faces, or geometric forms, is now being investigated.

As final points of discussion, the results of the present experiments will be related to findings reported by Graboi (1972) from a visual search experiment, and to findings reported by Ross (1970) from an itemrecognition experiment.

Graboi's (1971) visual search experiment was quite similar in design to that used with the new group in Experiment 2. Subjects were given prolonged practice searching for surnames, where positive sets were of Sizes $1,3,5$, and 7 , and nested. Negative items were 50 surnames, which were never used as positive items during the practice phase. Scanning time decreased with practice, and the set-size effect diminished, but was not eliminated. In one of the manipulations following practice, the positive items were replaced with "novel" items. Search times increased and the effect of set size increased markedly. This study has recently been cited by Corballis (1975) as showing that "The effect of practice on the slope of the search function or RT function appears to be specific to the particular memory sets that the Ss practice with" (p.600). It is most likely that the contradictory results obtained in the seemingly analogous designs in a visual search task and an item-recognition task are attributable to one or more critical differences in procedures. In this particular case, those differences seem quite obvious. On the transfer days, in Graboi's experiment, the items composing the highly practiced nested positive sets were deleted from the stimulus pool, and the novel positive sets were composed of items which had appeared as negative items throughout the practice sessions. Therefore, the so-called novel items were not novel at all, because they had already attached to them a response opposite to the one called for on the transfer days. Further, the positive sets were nonnested. It is not made clear whether novel items not in the currentlybeing-tested positive set size were used as negative items. In any case, on Graboi's transfer days, the introduction of the novel items by definition introduced a change in the design from response consistency to response inconsistency, and from nested to nonnested sets. Because of these confoundings, it is impossible to know to which variable(s) the changes from practice to transfer days should be attributed.

Ross's (1970) subjects were given prolonged practice 
in an item-recognition task, using alphanumeric uppercase characters, under conditions of response consistency with nested positive sets of Sizes $1,2,4$, and 8 . Reaction times were shown to be linearly related to the $\log$ of positive set size and the slope of this function decreased to approximately half its initial value over the course of practice. Following the practice sessions, the assignment of items to sets remained the same, but the size of the test item and its case were altered. Slope values were virtually unchanged by these manipulations. Thus, whatever was learned during practice transferred to the practiced characters even though they were presented in a different size and case, suggesting that the decision process involved in classification is, or at least can be, independent of the physical form of the practiced items. The experiments reported in the present paper may be seen as extensions of the Ross experiment; extensions which lead to the conclusion that whatever was learned during practice transferred to unpracticed characters. This finding suggests that for this construction of the task, after practice, the decision process involved in classification is independent of the identity of the stimulus as well.

\section{REFERENCES}

Briggs, G. E., \& Blaha, J. Memory retrieval and central comparison times in information processing. Journal of Experimental Psychology, 1969, 79, 395-402.

Burrows, D., \& Murdock, B. B., JR. Effects of extended practice of high-speed scanning. Journal of Experimental Psychology, 1969, 82, 231-237.
Corballis, M. C. Access to memory: An analysis of recognition times. In P. M. A. Rabbitt \& S. Dornic (Eds.). Attention and performance $V$. London: Academic Press, 1975. Pp. 591-612.

Grabor, D. Searching for targets: The effects of specific practice. Perception \& Psychophysics, 1971, 10, 300-304.

KRISTOFFERSON, M. W. Effects of practice on characterclassification performance. Canadian Journal of Psychology, 1972, 26, 54-60. (a)

Kristofferson, $M$. W. When item recognition and visual search functions are similar. Perception \& Psychophysics, 1972, 12, 379-384. (b)

NiCkERson, R. S. Response times with a memory-dependent decision task. Journal of Experimental Psychology, 1966, 72, 761-769.

Ross, J. Extended practice within a single-character classification task. Perception \& Psychophysics, 1970, 8, 276-278.

Simpson, P. J. High-speed memory scannings: Stability and generality. Joumal of Experimental Psychology, 1972, 96, 239-246.

SternberG, S. High-speed scanning in human memory. Science, 1966, 153, 652-654.

STERnberg, S. Memory-scanning: Mental processes revealed by reaction-time experiments. American Scientist, 1969, 57, 421-457.

\section{NOTE}

1. Details of these analyses are available from the author upon request.
(Received for publication April 5, 1976; revision accepted September 20,1976.) 